\title{
Logical foundation of fuzzy preferential systems with application to the electre decision aid methods
}

\author{
R. Bisdorff \\ CRP - Centre Universitaire, 162a, avenue de la Faïencerie, L-1511 Luxembourg
}

\begin{abstract}
In this paper we introduce split truth/falseness semantics for a multi-valued logical processing of fuzzy preference modelling. Our approach takes as starting point the standard framework of fuzzy outranking relations as proposed by the French multi-criteria decision aid community. Formal links between a given relational credibility calculus and associated truth polarization techniques will be discussed. The main result is the establishment of a multi-valued logical framework which allows us to naturally postpone any necessary defuzzification step to the end of the decision problem.
\end{abstract}

\section{Scope and purpose}

In this paper we propose an innovative multi-valued logical framework for processing fuzzy preferential information as is common in the context of multi-criteria preference modelling. Our results concern a methodological and computational enhancement of the Electre decision aid methods as promoted by the French school of decision aid around the work of B. Roy and D. Bouyssou (Roy. Méthodologie Multicritère d'Aide à la Décision. Paris: Economica, 1985; Roy and Bouyssou. Aide Multicritère à la Décision: Methodes et cas. Paris: Economica, 1993). The main achievement concerns the implementation of a logically coherent defuzzification technique in order to make commute logical reasoning and defuzzification of preferential information. (C) 2000 Elsevier Science Ltd. All rights reserved.

Keywords: Fuzzy preference modelling; Multi-criteria decision support; Electre decision aid methods

\section{Introduction}

A close look at the logical construction of Bernard Roy's approach to solve multi-criteria decision problems in the context of his Electre methods (see Roy [1] and Roy and Bouyssou [2])

E-mail address: bisdorff@crpcu.lu (R. Bisdorff) 
has convinced us that the operational essence of his decision-aid methodology lies in the clear logical distinction between the credibility calculus underlying the construction of relational indexes such as his concordance, discordance and outranking indexes, and the relational preference propositions we may induce from them. Indeed, the careful defuzzification step through $\lambda$-cuts on different levels $(\lambda \in[0,1])$, which projects multi-valued credibility values to their corresponding logical counterpart, namely truthfulness of a given preferential situation, is of crucial importance in his various approaches.

On the other hand, the fuzzy preference modelling community (see a.o. Fodor and Roubens [3], De Baets et al. [4]) is trying to do without this operational distinction by immediately formulating truth assumptions on multi-valued preference propositions, an eventual defuzzification step being seen as not really necessary in a multi-valued logical approach. But negative formal results have shown that there is no easy isomorphism between such a multivalued logical denotation and adequate set theoretical characterizations of preference structures, as is common with crisp relations (see for instance Fodor and Roubens [3, Chapter 3, pp. 71-84]).

In this paper, we therefore propose a third approach in-between the two preceding ones; an approach based on the natural multi-valued generalization of the traditional link between a multivalued credibility calculus and the induced multi-valued truth calculus.

\section{From credibility to truthfulness of propositions}

In our multi-valued logical considerations, we therefore make a clear semantic distinction between some underlying credibility calculus qualifying the truthfulness of given atomic propositions, and the corresponding induced multi-valued truthfulness of logical expressions involving these propositions.

\subsection{Basic credibility calculus}

Definition 1. Let $\mathscr{P}$ represent a set of atomic propositional formulas $p$ to which we may associate a finite rational degree of credibility $r(p) \in[0,1]$ with respect to the potential truthfulness of the proposition $p$. If $r(p)=1$, proposition $p$ is perceived as certainly true, and if $r(p)=0$, proposition $p$ is perceived as certainly false. The complete ordered finite set of involved credibility values is denoted $V$. The underlying order is denoted $(V, \leqslant)$, where $\leqslant$ denotes a complete, reflexive, antisymmetric and transitive ordering.

In multi-criteria decision aid practice, degrees of credibility associated with a given basic preferential proposition are generally expressed as 2-digit percentages in the following way: "alternative $a$ is perceived as at least as good as alternative $b$ with a credibility of $75 \%$ ".

Definition 2. Let $(\mathscr{P}, r)$ be a set of atomic propositions $p$ associated with corresponding degrees of credibility $r: \mathscr{P} \rightarrow V$. Let $\neg, \vee, \wedge$ and $\rightarrow$ denote, respectively, negation, disjunction, conjunction and implication. The set $\mathscr{E}$ of all well-formulated finite expressions will be generated inductively from 
the following grammar:

$$
\begin{aligned}
& \forall p \in \mathscr{P}: p \in \mathscr{E}, \\
& \forall x, y \in \mathscr{E}: \neg x|(x)| x \vee y|x \wedge y| x \rightarrow y \in \mathscr{E} .
\end{aligned}
$$

The unary operator $\neg$ has a higher precedence in the interpretation of a formula, but generally we use bracketing parentheses to control the application range of a given operator and thus to make all formulas have unambiguous semantics.

We extend the credibility calculus on such logical expressions in the following way:

Definition 3. Let $\mathscr{E}$ be a set of well formulated expressions based on $\mathscr{P}$. $\forall x, y \in \mathscr{E}$ :

$$
\begin{aligned}
& r(\neg x)=1-r(x), \\
& r(x \vee y)=\max (r(x), r(y)), \\
& r(x \wedge y)=\min (r(x), r(y)), \\
& r(x \rightarrow y)=1 \Leftrightarrow r(x) \leqslant r(y) .
\end{aligned}
$$

From the inductive definition of our well formulated expressions, we are thus able to compute the credibility of any such formula in what we call a symmetric evaluation domain $\mathscr{L}=\left(V, \leqslant, \neg, \min , \max , \rightarrow, 0, \frac{1}{2}, 1\right)$. The negation operator ' $\neg$ ' implements a strict anti-tonic bijection with credibility $\frac{1}{2}$ acting as negational fix-point. Classic min and max operators capture credibilities of conjunction respective disjunction of formulas. For the sake of simplicity in the scope of this paper, we use a partial crisp implementation of $\mathscr{L}$-valued implication ' $\rightarrow$ ', which we shall call for short ordinal implication. ${ }^{1}$ Finally, we denote the couple $(\mathscr{E}, r)$ as $\mathscr{E}^{\mathscr{L}}$ and simply speak of $\mathscr{L}$-valued expressions in the rest of the paper.

Two such special $\mathscr{L}$-domains are worth noticing here: - first, the three-valued domain $\mathscr{L}_{3}=\left(\left\{0, \frac{1}{2}, 1\right\}, \leqslant, \neg, \min , \max , \rightarrow, 0, \frac{1}{2}, 1\right)$ giving in fact a three-valued type of logic; and - secondly, the classical bi-valued (degenerated) domain $\mathscr{B}=(\{0,1\}, \leqslant, \neg$, min, $\max , \rightarrow, 0,1)$ isomorphic to classical Boolean logic.

Knowing the credibility of a given $\mathscr{L}$-valued expression, we are now able to induce its supposed truthfulness.

\subsection{Defining the truthfulness of an $\mathscr{L}$-valued expression}

In classical bi-valued logic, it is usual to work syntactically only on the truth point of view of the logic, the falseness point of view being redundant through the coercion to the excluded middle. For instance, writing " $(\mathrm{a}, \mathrm{b}) \in R$ " implicitly means assuming that this proposition is actually true and its negation false, otherwise we would write “ $(a, b) \notin R$ ". We will also rely syntactically on such an implicit truth point of view and always denote the truthfulness possibly induced from the

\footnotetext{
${ }^{1}$ Other models such as the Goedel or the implication would also be applicable, but these implementations make necessary to specify an algebraic refinement tukasiewicz of the underlying $\mathscr{L}$-algebra. For instance, a tukasiewicz logic would require additive properties on $V$. In fact, we do not need a more precise operational definition in what follows.
} 
underlying credibility calculus through a $\mu$ operator $^{2}$ acting as domain restriction on the credibility operator $r$.

Definition 4. Let $x \in \mathscr{E}^{\mathscr{L}}$ be an $\mathscr{L}$-valued expression associated with credibility $r(x)$ :

$$
\mu(x)=r(x) \Leftrightarrow r(x) \geqslant r(\neg x) .
$$

Truthfulness of a given expression $x$ is only defined in case the expression's credibility $r(x)$ exceeds the credibility $r(\neg x)$ of its contradiction $\neg x$. From our definition, it follows immediately that our induced $\mathscr{L}$-valued truth calculus is complete on every set $\mathscr{E}^{\mathscr{L}}$ of well formulated $\mathscr{L}$-valued expressions, that is $\forall x \in \mathscr{E}^{\mathscr{L}}$ : either $\mu(x)$ or $\mu(\neg x)$ is defined. We say for short that any expression $x \in \mathscr{E}^{\mathscr{L}}$ is $\mathscr{L}$-true or $\mathscr{L}$-false.

To illustrate our approach, let us now look at what happens with the truthfulness of certain classical tautologies or antilogies in our $\mathscr{L}$-valued framework. For instance, truthfulness of the tautology $(p \vee \neg p)$, given by $\mu(p \vee \neg p)$, is always defined, as $\max (r(p), 1-r(p)) \geqslant \frac{1}{2}$ in any case. Tautological $\mathscr{L}$-valued propositions thus appear as being $\mathscr{L}$-true in any case. Therefore, we call them $\mathscr{L}$-tautologies. On the other hand, as truthfulness of the antilogy $(p \wedge \neg p)$, we only obtain the following undetermined truth value: $\mu(p \wedge \neg p)=\frac{1}{2} \Leftrightarrow p=\neg p=\frac{1}{2}$. Truthfulness of such a proposition is undefined otherwise. But its contradiction is $\mathscr{L}$-true, i.e. $\mu(\neg(p \wedge \neg p))$ is always defined. Therefore, we call such propositions $\mathscr{L}$-antilogies. As a main result of our construction, we recover in this sense all classical tautologies and antilogies as particular limit case $\mathscr{E}^{\mathscr{B}}$ if we reduce our $\mathscr{L}$-valued credibility calculus to a bi-valued one.

Indeed, let us investigate an implicative $\mathscr{L}$-tautology such as modus ponens for instance.

If we take the classical negative (Kleene-Dienes) definition of the implication, i.e. falseness of the conjunction of $p$ and $\neg q$, we obtain

$$
\min (r(p), \max (1-r(p), r(q))) \geqslant \frac{1}{2} \Rightarrow r(q) \geqslant \frac{1}{2},
$$

i.e. the following $\mathscr{L}$-tautology: $p$ and $p \Rightarrow q$ being conjointly $\mathscr{L}$-true always implies $q$ being $\mathscr{L}$-true. If we stay with our ordinal implication, ${ }^{3}$ we obtain:

$$
\min (r(p), r(p \rightarrow q)) \geqslant \frac{1}{2} \Rightarrow r(q) \geqslant r(p) \geqslant \frac{1}{2},
$$

i.e. again an $\mathscr{L}$-tautology.

At this point, one may wonder if our $\mathscr{L}$-logic does not appear as a structure isomorphic to classical bi-valued Boolean logic. But this is not the case, as the natural categorical limit $\mathscr{L}$-algebra is given by an $\mathscr{L}_{3}$ logic. But we can force our $\mathscr{L}$-valued truth calculus to take a bi-valued Boolean logic $\mathscr{B}$ as limit, by explicitly excluding the contradictional fix-point from the set of possible

\footnotetext{
${ }^{2}$ In fuzzy set theory, the $\mu$ operator generally denotes a fuzzy membership function. We here choose the same $\mu$ symbol on purpose as our main $\mathscr{L}$-valued formulas mostly concern such $\mathscr{L}$-valued characteristic functions.

${ }^{3}$ It may be interesting at this point to notice that both possible definitions of implication in our $\mathscr{L}$-valued credibility calculus, being equivalent in the Boolean case, are not at all semantically equivalent in our case. Indeed, the negative definition is only applicable in a logic where a complementary negation is semantically equivalent with a contradiction. Here this is not the case, and a specific definition of $\mathscr{L}$-valued consequent calculus must be given under the form of this ordinal implication.
} 
credibility values; thus making it impossible to stay logically neutral in the underlying credibilities and hence also in our truthfulness assumptions.

Applying this system to fuzzy preference modelling now, we obtain the following basic semantics.

\section{Basic semantics of $\mathscr{L}$-valued preferential systems}

As initially stated, we would like to apply our credibility and truth calculus to decision aid problems in a fuzzy preference modelling context.

\subsection{The basic $\mathscr{L}$-reflexive outranking relation}

The initial set $\mathscr{P}$ of atomic propositions is given by a fundamental $\mathscr{L}$-valued binary relation $R$, defined on a finite set $A$ of alternatives, with the following general semantics:

\section{Definition 5.}

$\forall a, b \in A:(a \mathrm{R} b) \equiv$ " $a$ more or less outranks $b$ ".

This outranking relation may also be interpreted as "being at least as good as".

In our case, we suppose that relation $\mathrm{R}$ is associated with given rational degrees of credibility modelled as an outranking index $r: A \times A \rightarrow V$, i.e. an $\mathscr{L}$-valued characteristic function on the support $A \times A$ of relation $\mathrm{R}$.

Normally, one is tempted to take this outranking index directly as the associated fuzzy truth value of the proposition "a more or less outranks $b$ ". ${ }^{4}$ But, in accordance with our split truth/falseness semantics, we restrict the induced truthfulness $\mu(a, b)$ assignment as follows:

\section{Definition 6.}

$$
\forall a, b \in A: \mu(a \mathrm{R} b)=r(a, b) \Leftrightarrow r(a, b) \geqslant 1-r(a, b) .
$$

The proposition " $(a \mathrm{R} b)$ " is more or less true if and only if the credibility $r(a, b)$ associated with $(a \mathrm{R} b)$ is greater or equal to the credibility $1-r(a, b)$ associated with its contradiction $(a \mathbb{R} / b)$. Similarly, we consider the contradicted proposition to be more or less true in the following sense:

\section{Definition 7.}

$$
\forall a, b \in A: \mu(a \mathbb{R} b)=1-r(a, b) \Leftrightarrow 1-r(a, b) \geqslant r(a, b) .
$$

As a consequence, we obtain in our $\mu^{\mathscr{L}}$-semantics the following equivalent statements:

\footnotetext{
${ }^{4}$ See for instance the definition of valued binary relations as proposed by Fodor and Roubens in [3, Section 2.2, p. 42].
} 


\section{Proposition 8.}

$\forall a, b \in A: \mu(a \mathbb{R} b)$ is defined $\Leftrightarrow r(a, b) \geqslant \frac{1}{2}$,

$\forall a, b \in A: \mu(a \mathbb{R} b)$ is defined $\Leftrightarrow 1-r(a, b) \geqslant \frac{1}{2}$.

Proof. Indeed, the strong negational contradiction $(1-r)$ installs a functional link between both truthfulness assumptions through the strict antitonic bijection which we have chosen as its algebraic implementation in the underlying credibility calculus. The unique contradictional fix-point, i.e. the logically undetermined truth value $\frac{1}{2}$, thus represents precisely the semantic bifurcation point between more or less truthfulness or falsefulness ${ }^{5}$ of the $\mathscr{L}$-valued relational propositions.

In short, for any $\mathscr{L}$-valued relation $\mathrm{R}^{\mathscr{L}}$, we denote $\mu\left(\mathrm{R}^{\mathscr{L}}\right)$ the subset of all pairs $(a, b) \in \mathrm{R}^{\mathscr{L}}$ such that $r(a, b) \geqslant \frac{1}{2}$. In general, we denote as $\mathscr{L}$-subset $\mu(S)$, the subset of $\mathscr{L}$-true characterized elements in a set $S$.

Let us now define general $\mathscr{L}$-valued preferential systems.

\section{2. $\mathscr{L}$-preference, $\mathscr{L}$-indifference and $\mathscr{L}$-incomparability}

On the basis of our general outranking relation $R$, it is now possible to define adequate preference, indifference and incomparability relations, denoted respectively as $\mathrm{P}, \mathrm{I}$ and $\mathrm{J}$. The Semantics of each relation is given as follows: $\forall a, b \in A$ :

$(a \mathrm{P} b) \equiv$ " $a$ is more or less preferred to $b "$,

$(a \mathrm{I} b) \equiv$ " $a$ is more or less indifferent to $b "$,

$(a \mathrm{~J} b) \equiv$ " $a$ is more or less incomparable to $b "$.

We can now define, on the basis of the $\mathscr{L}$-valued outranking index $r$, convenient $\mathscr{L}$-valued preference, indifference and incomparability indexes denoted, respectively, $p, i$ and $j$ and capturing the credibilities of these relational propositions. ${ }^{6} \forall a, b \in A$ :

$$
\begin{aligned}
& p(a, b)=\min (r(a, b), 1-r(b, a)), \\
& i(a, b)=\min (r(a, b), r(b, a)), \\
& j(a, b)=\min (1-r(a, b), 1-r(b, a)) .
\end{aligned}
$$

And again we can state that $\forall a, b \in A$ :

$$
\begin{aligned}
& \mu(a \mathrm{P} b)=p(a, b) \Leftrightarrow p(a, b) \geqslant \frac{1}{2}, \\
& \mu(a \mathrm{I} b)=i(a, b) \Leftrightarrow i(a, b) \geqslant \frac{1}{2}, \\
& \mu(a \mathrm{~J} b)=j(a, b) \Leftrightarrow j(a, b) \geqslant \frac{1}{2} .
\end{aligned}
$$

Let us now establish a first important result:

\footnotetext{
${ }^{5}$ To the classic opposition truth/falseness we add the fuzzy opposition truthfulness/falsefulness.

${ }^{6}$ We keep with the formalism introduced by Fodor and Roubens in [3, p. 71]
} 
Proposition 9. Let $\mu(\mathrm{P}), \mu(\mathrm{I})$ and $\mu(\mathrm{J})$ be the $\mathscr{L}$-subsets, i.e. the relational membership propositions being $\mathscr{L}$-true on the basis of the underlying corresponding relational credibility indexes: $p, i$ and $j$. Let $\mu\left(\frac{1}{2}\right)$ stand generically for the set of $\frac{1}{2}$-valued propositions in $\mathscr{E}^{\mathrm{R}}$. Then we have:

$$
\begin{aligned}
& \mu(\mathbf{P} \cup \mathbf{I})=\mu(\mathbf{R}), \\
& \mu(\mathbf{P} \cap \mathbf{I}) \subseteq \mu\left(\frac{1}{2}\right), \\
& \mu(\mathbf{P} \cap \mathbf{J}) \subseteq \mu\left(\frac{1}{2}\right), \\
& \mu(\mathbf{I} \cap \mathbf{J}) \subseteq \mu\left(\frac{1}{2}\right), \\
& \mu\left(\mathbf{P} \cup \mathbf{I} \cup \mathbf{P}^{-1}\right)=\mu\left(\mathbf{R} \cup \mathbf{R}^{-1}\right) .
\end{aligned}
$$

Proof. Indeed, for relation (18) we have $\mu(\mathbf{P} \cup \mathbf{I})=\{(a, b) \in A \times A \mid \max ((\min (r(a, b), 1-r(b, a))$, $\left.(\min (r(a, b), r(b, a))) \geqslant \frac{1}{2}\right\}$. This forces $r(a, b) \geqslant \frac{1}{2}$. And similarly, for relation (19) we have $\mu(\mathbf{P} \cap \mathbf{I})=\left\{(a, b) \in A \times A \mid \min \left(\left(\min (r(a, b), 1-r(b, a)),(\min (r(a, b), r(b, a))) \geqslant \frac{1}{2}\right\}\right.\right.$. This again forces $r(b, a)=1-r(b, a)=\frac{1}{2}$.

All other relations can be verified in the same straightforward way.

It is interesting to compare our positive result with the negative result obtained by Alsina in 1985. The demonstration, as stated by Fodor and Roubens (see [3, p. 73]) clearly shows, that the set theoretic conclusions in a strong symmetrical multi-valued logic must respect the underlying bi-fold semantics of truthfulness versus falsefulness as distributed by the contradictional fix-point $\frac{1}{2}$.

\section{Logical fuzzification and polarization: an adjoint pair}

The semantic difference between the logical fuzziness we introduce, and the standard fuzziness as modelled in fuzzy or multi-valued logic and/or in fuzzy set theory, lies - first in the operational distinction between the underlying propositional credibility calculus and the induced truthfulness calculus, and - secondly in the strict splitting of the credibilities into truthfulness supporting ones and falsefulness supporting ones, with a possible unique common intersection only in case of logical undeterminedness.

In this section we investigate more categorically the previously introduced semantics through the general techniques of fuzzification and defuzzification. First, we turn to logical fuzzification.

\subsection{Introducing logical fuzzification}

A common sense fuzzification of the classic bi-valued truth calculus consists in replacing the underlying bi-valued characteristic function with a multi-valued bounded real-valued characteristic function followed by the attempt to axiomatize such real-valued logical calculus in such a way to recover all known (and so generally accepted as useful) logical properties of the bi-valued case. In our opinion, this straightforward method suffers from two main drawbacks: - first, such simple multi-valued generalization of the bi-valued characteristic function is in contradiction with the necessarily split semantics of the implicitly modelled truth/falseness point of views and - secondly, 
Table 1

Example of fuzzy outranking relation

\begin{tabular}{lllllllll}
\hline$r$ & $a$ & $b$ & $c$ & $d$ & $e$ & $f$ & $g$ & $h$ \\
\hline$a$ & 1 & 0 & 0 & 0 & 0 & 0 & 0 & 0 \\
$b$ & 0.75 & 1 & 0.57 & 0.57 & 0.57 & 0.63 & 0.57 & 0.57 \\
$c$ & 0.70 & 0.46 & 1 & 0.64 & 0.64 & 0.46 & 0.46 & 0.64 \\
$d$ & 0.62 & 0.22 & 0.22 & 1 & 0.60 & 0.22 & 0.22 & 0.60 \\
$e$ & 0 & 0 & 0 & 0 & 1 & 0 & 0 & 0.57 \\
$f$ & 0 & 0 & 0 & 0 & 0 & 1 & 0 & 0 \\
$g$ & 0 & 0 & 0 & 0 & 0 & 0.63 & 1 & 0 \\
$h$ & 0 & 0 & 0 & 0 & 0 & 0 & 0 & 1 \\
\hline
\end{tabular}

some of the structural properties of a bi-valued logical framework appear as degenerated limit properties, impossible to observe in the general case without precisely questioning this generalization again. Our reason for thinking so is given, not so much by the axiomatic difficulties of the standard attempt, but rathermore by the dubious operational properties of the corresponding standard defuzzification technique, denoted in the fuzzy literature as $\lambda$-cuts, where $\lambda \in V$ represents the level of credibility at which a given proposition is taken to be true or not.

To illustrate the difficulty, we consider the definition of cut relations as discussed in Fodor and Roubens (see [3, p. 45]). Let us take a fuzzy outranking index, as shown in Table 1. For a given $\lambda \in V$, Fodor and Roubens propose to consider the relation $\mathrm{R}_{\lambda}$ as being defined as the set of pairs $(a, b) \in A \times A$ such that $r(a, b) \geqslant \lambda$. What has escaped the authors' minds is the fact that a cut technique is necessarily either truth or falseness oriented and that the resulting crisp or bi-valued relation is, in their case, taken from the truth point of view. But this point of view cannot, in a standard logical frame, where a contradiction principle must exist, be separated from the complementary falseness point of view. Stating that for instance there exists a 0.20 -cut relation on the index $r$ as shown in Table 1 implicitly implies, in case we envisage a credibility calculus with a strong negation, an asymmetric treatment of truthfulness versus falsefulness. Indeed, we would consider proposition " $(d \mathrm{R} b)$ " to be true, as $r(d, b)=0.22>0.20$ and conjointly proposition " $(d \mathbb{R} b)$ " to be also true as $1-r(d, b)=0.8>0.2$, and thereby we would introduce logical nonsense. But if we solely envisage $\lambda$-cut relations with $\lambda \geqslant \frac{1}{2}$, we may obtain possibly correct split truthfulness versus falsefulness semantics. Indeed, consider again the same pair $(d \mathrm{R} b)$. This time, we conclude that $(d \mathbb{R} b)$ as $r(d, b)=0.20<0.50$, this result being coherent with the fact that $1-r(d, b)=0.80>0.50$.

Two lessons are to be learned from this example. First, every logical truth calculus conjointly addresses a mirrored falseness calculus through the necessary existence of a contradiction principle. Secondly, fuzzification models are not to be separated from intended defuzzification or logical polarization techniques, as defuzzification necessarily projects fuzziness into the split complementary semantics of truthfulness versus falsefulness.

The above considerations give rise to the following question: why does Boolean calculus nevertheless work, although it does not use such a split semantics?

In this specific case, the reason for this simplification lies in the operational restriction to two extreme truth values $\{0,1\}$, with implied coercion to the excluded middle, which simplifies the 


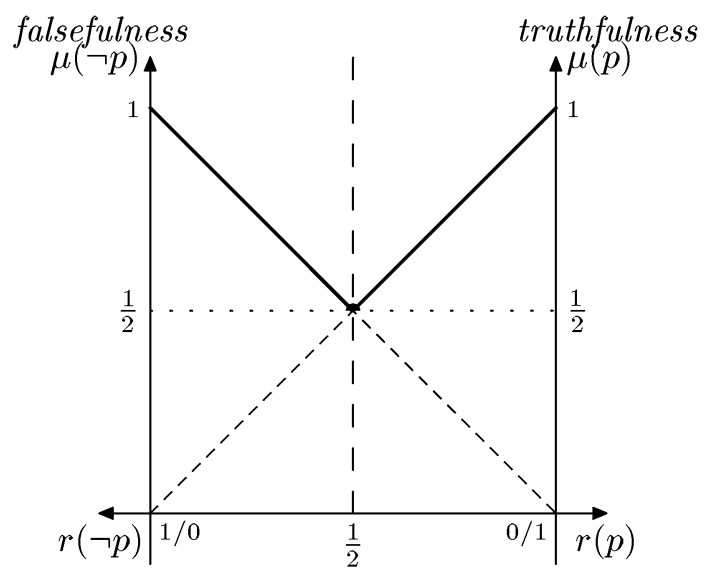

Fig. 1. Split truth/falseness semantics.

underlying logical algebra to a point making the necessity to separate truthfulness values from falsefulness values disappear. Indeed, both points of view are completely determined one by the other, as no intermediate position is allowed. As soon as intermediate logical positioning is allowed, the necessity also appears to algebraically separate the truthfulness point of view from the falsefulness point of view and eventually to introduce a third term, the necessarily unique common truth/falseness point of view: logical undeterminedness. It appears from the algebraic framework that, in case we envisage a strong negational contradiction, this logical undeterminedness resides in the necessarily unique negational fix-point, i.e. the truthfulness/falsefulness value $\frac{1}{2}$ marking the common border line between appearing truthfulness versus appearing falsefulness (see Fig. 1).

To illustrate our approach, we consider a simple majority voting procedure generating atomic propositions with respective credibilities reflecting the positive or negative results of the individual voting. A proposition is true from the moment it gets more than half of the possible votes and it is false elsewhere. The exact result models the greater or lesser truthfulness or falsefulness of the result. But no proposition may be conjointly accepted and rejected unless it gets exactly half of the votes. In practice, this blocking case is sometimes avoided either by considering only odd numbers of possible voters or by a specific de-blocking procedure like for instance counting the chairman's vote for double in this situation.

One may question the simple majority rule. Why is it just half and not lesser of the votes that distribute truthfulness and falsefulness? Mostly for practical reasons: if less than $\frac{1}{2}$ of positive votes would be sufficient to accept a proposition, a proposition could in practice be both accepted and rejected, which would produce practical (logical) nonsense. On the contrary, higher qualified majority rules are very often used in practice and especially for important decisions. How can we cope with this issue in our logical framework?

\subsection{On natural logical polarization}

What we are looking for is a defuzzification or logical polarization operator denoted $\pi$, similar to the $\lambda$-cut but compatible with our split truth/falseness semantics. 
In Bisdorff and Roubens [5], we have for the first time introduced such a polarization operator $\pi_{\beta}$, which we named the $\beta$-cut. ${ }^{7}$

Definition 10. Let $\mathscr{E}^{\mathscr{L}}$ be a set of logical expressions associated with credibility $r: \mathscr{E}^{\mathscr{L}} \rightarrow V$, where $\mathscr{L}=\left\{V, \geqslant, \neg\right.$, min, $\left.\max , \rightarrow, 0, \frac{1}{2}, 1\right\}$ underlies algebraically the propositional credibility calculus. Let $\pi_{\beta}$ represent a logical polarization operator $\pi_{\beta}: \mathscr{E}^{\mathscr{L}} \rightarrow \mathscr{E}^{\mathscr{L}_{3}}$ defined as follows: $\forall x \in \mathscr{E}^{\mathscr{L}}$ and $\left.\forall \beta \in] \frac{1}{2}, 1\right]$ :

$$
\pi_{\beta}(r(x))=\left\{\begin{array}{l}
1 \Leftrightarrow r(x)>\beta, \\
0 \Leftrightarrow r(x)<1-\beta, \\
\frac{1}{2} \Leftrightarrow 1-\beta \leqslant r(x) \leqslant \beta .
\end{array}\right.
$$

That this polarization operator $\pi_{\beta}$ indeed satisfies our formal expectations may be summarized by stating the following theorem:

Theorem 11. Let $\mathscr{E}^{\mathscr{L}}$ be a set of $\mathscr{L}$-valued expressions. Let $\mu$ denote our truthfulness operator, $\pi_{\beta}$ the $\beta$-cut polarization operator and $\pi_{1 / 2}$ the median $\beta$-cut operator. The following categorical equations are verified:

$$
\begin{aligned}
& \mu \circ \pi_{1 / 2}=\pi_{1 / 2} \circ \mu, \\
& \mu \circ \pi_{\beta} \supseteq \pi_{\beta} \circ \mu .
\end{aligned}
$$

Proof. Considering Eq. (23), we must show that the $\pi_{1 / 2}$ operation is a natural transformation of $\mathscr{L}$-valued expressions with respect to our $\mu$ operator. Let us first consider disjunction (respectively, conjunction) of two $\mathscr{L}$-valued expressions $x, y \in \mathscr{E}^{\mathscr{L}}$. Let $\pi_{1 / 2}(x)$ and $\pi_{1 / 2}(y)$ be the associated $\frac{1}{2}$-cut propositions. $\pi_{1 / 2}(\mu(x \vee / \wedge y))=\max / \min (r(x), r(y)) \Leftrightarrow\left(r(x) \geqslant \frac{1}{2}\right) \vee / \wedge\left(y \geqslant \frac{1}{2}\right) \Leftrightarrow \mu\left(\pi_{1 / 2}(x) \vee \pi_{1 / 2}(x)\right)=$ $\max / \min (r(x), r(y))$. The same straightforward argument applies to the two other logical operators in $\mathscr{L}$, i.e. contradiction and ordinal implication. Considering now Eq. (24), it is precisely the ordinal implication that appears as more restrictive than a negative $\mathscr{L}$-valued Kleene-Dienes implication, thus generally making the $\pi_{\beta}$ operator only semi-natural for the $\mu$ transform (see Bisdorff and Roubens [5]).

It is clear that the demonstration above is highly dependent on the choice of the $\mathscr{L}$-algebra and it would be interesting to furthermore characterize naturally defuzzifiable credibility calculi in the sense above.

To illustrate now the use of our logical framework in the context of a given decision aid methodology, we discuss in a last section, Electre choice recommendations that can be constructed from a given outranking relation (see Roy and Bouyssou [2]).

\footnotetext{
${ }^{7}$ The name we gave this logical polarization operator is derived from the fact that the standard $\lambda$-cut is sometimes also named $\alpha$-cut.
} 


\section{Application to the Electre choice recommendation method}

In order to illustrate the methological enhancement that allows our $\mathscr{L}$-logic framework, we discuss in this section a didactical ${ }^{8}$ choice problem proposed by Bernard Roy at the 41 st meeting of the EURO working group MCAD on "Multi-criteria Aid for Decisions" in Lausanne, March 1995 on the occasion of a lecture by Marc Roubens on Leonid Kitaïnik's [6] work about fuzzy kernels.

We suppose that a decision-maker has to make a unique choice from a set of three R\&D projects represented as $A=\{\mathbf{a}, \mathbf{b}, \mathbf{c}\}$. We furthermore assume that a given decision aid analysis has eventually led to the following outranking index $r$ on $A \times A$ :

(1) a outranks b with credibility $=0 \%$;

(2) a outranks c with credibility $=100 \%$;

(3) b outranks a with credibility $=70 \%$;

(4) b outranks c with credibility $=100 \%$;

(5) $\mathbf{c}$ outranks $\mathbf{b}$ and $\mathbf{a}$ with credibility $=70 \%$.

The following outranking graph can be associated with the outranking index $r$ :

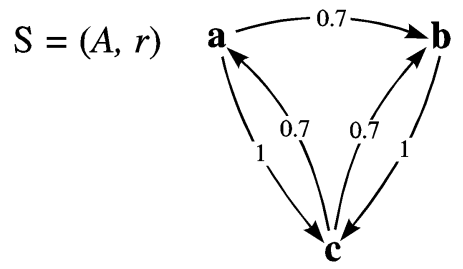

Assuming general semantics of the outranking relation given by "to be at least as good as", what $\mathrm{R} \& \mathrm{D}$ project could we advise the decision-maker to choose?

Following Roy and Bouyssou [2], solving this choice problem requires the construction of a unique possibly univocal selection from set $A$. However, two possibilities of solving the decision problem arise: - first, defuzzifying the credibilities and solving the resulting crisp relational problem, a solution traditionally followed by Roy; - secondly, solving the fuzzy relational problem and defuzzifying the result thus obtained, a solution proposed by Bisdorff and Roubens $[5,7]$.

\subsection{Solving the defuzzified decision problem}

In his family of ELECTRE I methods, Roy proposes to defuzzify the given outranking graph $S$, by considering plausible (most robust) $\lambda$-cuts. The idea is to replace the original fuzzy out-ranking relation $S$ by a crisp relation $S_{\lambda}$, which appears as most robust.

What possible defuzzification can we propose for the Lausanne example?

\footnotetext{
${ }^{8}$ Some readers might be disappointed not to find here the application to a real-life decision aid problem. But we think that the above chosen didactical example better fits in with our references to the corresponding work of Roy and Bouyssou (see [2]). The interested reader may find a real-life application of our approach in [8].
} 


\subsubsection{1st solution}

Consider all fuzzy out-ranking relations as sufficiently credible. This gives the following graph:

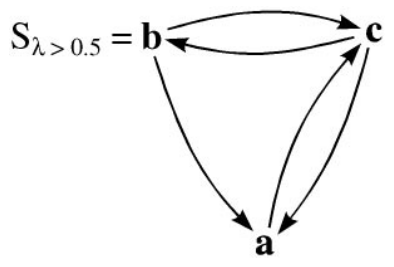

All credibilities values 0.70 are changed to 1 and we face a case explicitly cited by Roy and Bouyssou [2, p. 336] where the authors propose to choose alternative $\mathbf{b}$ from among the subset of optimal alternatives $\{\mathbf{b}, \mathbf{c}\}$ on the basis of an argument that $\mathbf{c}$ is perceived to be slightly lower than b with respect to its comparison with a (“c se compare moins bien à a que b").

\subsubsection{2nd solution}

Consider the more or less credible out-rankings as not convincing the decision maker; the credibility values 0.70 are changed to 0 by for instance using a corresponding 0.75 -cut.

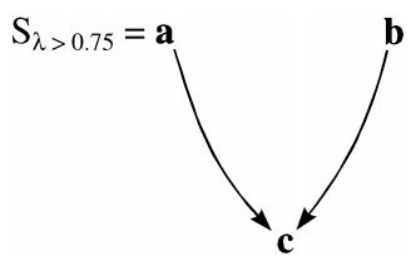

This time, the subset of optimal solutions is given by $\{\mathbf{a}, \mathbf{b}\}$, without any possibility to prefer either $\mathbf{a}$ or $\mathbf{b}$. But we notice here the asymmetric defuzzification the $\lambda$-cut approach introduces; thus making the second solution appear as not as robust as the first one, a consequence not of the problem as it is given, but an indirect consequence of the unnatural defuzzification technique applied. Indeed, in the first case, we project $70 \%$ credibility to credibility $100 \%$, and in the second case, we project credibility $70 \%$ to credibility $0 \%$.

The standard $\lambda$-cut defuzzification technique is not coherently balanced and we propose to change this technique by considering the above introduced $\beta$-cut technique, relying on a symmetric treatment of more or less truthfulness against more or less falsefulness.

If we consider, as in solution 1 above, a very low level of confirmness for the $\beta$-cut defuzzification $(\beta>50 \%)$, we in fact recover the same solution as above associated with the same robustness. ${ }^{9}$

But when we consider $\beta$-cut defuzzification at level $\beta>75 \%$ for instance:

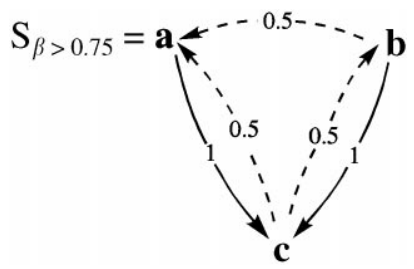

\footnotetext{
${ }^{9}$ It is worth noticing that indeed for an out-ranking relation not containing any logically undetermined truth value, median $\lambda$ - and $\beta$-cut defuzzification give identical results (see Bisdorff and Roubens [5]).
} 
we notice the robustness of this defuzzification as being most convincing, in the sense that, in this example, we only slightly fuzzify the originally given outranking index by changing $70 \%$ credibilities to $50 \%$ ones.

But we apparently loose an intuitive selection procedure from such a three-valued polarized relation, as the selection of a given alternative $x$ may become certainly true, certainly false or perhaps logically undetermined.

However, careful observation shows that two equivalent $\mathscr{L}$-subsets for recommendation are possible: $\{\mathbf{a}(1), \mathbf{b}(0.5), c(0)\}$ and $\{\mathbf{a}(0.5), \mathbf{b}(1), c(0)\}$, either certainly select alternative $a$, certainly reject alternative $c$ and leave the choice of alternative $b$ undetermined, or certainly choose alternative $b$, certainly reject alternative $c$ again and leave the choice of alternative $a$ undetermined this time.

Further empirical investigation should provide complementary arguments to eventually discriminate between the actually equivalent alternatives.

As a conclusion, we can see that this first approach, independently of the applied defuzzification technique, relies heavily on the outcome of the defuzzification step for the discussion of the actual outcome of the solving step. Therefore, with Theorem 11 in mind we advocate a second approach: - first, solving the fuzzy decision problem and then only — polarizing the potentially fuzzy result obtained.

\subsection{Solving the fuzzy decision problem and defuzzifying the result}

In [5,7], we therefore propose to solve the given problem with the help of a corresponding $\mathscr{L}$-valued kernel construction. Let us briefly recall that a dominant kernel $K^{\mathrm{R}}$ on an outranking relation $R$ is an interior and exterior stable subset of $A$ with respect to $R$.

In the case of an $\mathscr{L}$-valued relation, we can naturally (in a categorical sense) extend the crisp dominant kernel concept to our $\mathscr{L}$-valued semantics and speak of dominant $\mathscr{L}$-kernels, that is $\mathscr{L}$-true selected interior and exterior stable subsets of $A$ (see Bisdorff and Roubens [5]).

In the case of the given outranking relation $S$ above, we would get the following $\mathscr{L}$-valued kernel solutions:

$$
K_{1}^{\mathrm{S}}=\{a(30 \%), \mathbf{b}(100 \%), c(0 \%)\},
$$

and

$$
K_{2}^{\mathrm{S}}=\{a(30 \%), b(30 \%), \mathbf{c}(70 \%)\} .
$$

If we apply our truth operator $\mu$ to this result we obtain:

(1) b certainly gives a unique choice candidate (credibility $=100 \%$ );

(2) $\mathbf{c}$ more or less gives a unique choice candidate (credibility $=70 \%$ );

If we now apply our defuzzification operator $\pi_{\beta}$ to this result, we obtain alternative $b$, as well as alternative $c$, as potential unique choice candidates in the first case, i.e. when all $\mathscr{L}$-true credibilities are considered:

$$
K_{1 \beta>0.5}^{S}=\{a(0 \%), \mathbf{b}(100 \%), c(0 \%)\},
$$

and

$$
K_{2 \beta>0.5}^{S}=\{a(0 \%), b(0 \%), \mathbf{c}(100 \%)\} .
$$


Following Theorem 11 again, we naturally recover here the same result as in the former approach. Better, we now get a formal argument for the intuitive statement that alternative $b$ appears to be a more robust recommendation; the corresponding credibility of $(100 \%)$ is certainly higher than the credibility of $(70 \%)$, concerning the recommendation of alternative $c$.

If we now consider only credibilities above $75 \%$ as to be accepted as representing 'certainly true', we naturally stay with our unique best choice candidate, namely alternative $b$. And indeed, from the former approach, we already know that alternative $b$ remains a certainly credible choice recommendation even under this more pessimistic view. Recalling Eq. (24) of Theorem 11 we notice that we partly recover the set of $\beta$-cut solutions which we got previously.

Finally, compared to the former approach, where the problem is solved after multiple possible initial defuzzifications, with the operational difficulty of judging which is the best one, we are now confronted in our approach with a unique defuzzification, solely depending on the decisionmaker's conviction to accept the underlying basic credibilities as sufficient for supporting truth of the choice recommendations.

\section{Conclusion}

In this paper, we have discussed the close link between on the one hand, the treatment of logical fuzziness through a corresponding credibility calculus, associated with relational propositions and, on the other hand, the careful defuzzification that we must operate in order to conclude coherently on the nature of truth or falseness which this underlying credibility calculus projects on the relational propositions.

The Main result appears to be the coherent treatment of logical fuzziness with respect to classical Boolean logic by restricting our truth constructions to split truth/falseness semantics. Finally, a natural defuzzification technique ( $\beta$-cuts) has been introduced, thus allowing us to make defuzzification and solving of the decision problem commute. This operational result allows us to keep necessary defuzzification solely under the control of the actual decision-maker himself, which means eventually a better reception of the given decision recommendation, i.e. of the decision aid.

\section{References}

[1] Roy B. Méthodologie Multicritère d'Aide à la Décision. Paris: Economica, 1985.

[2] Roy B, Bouyssou D. Aide Multicritère à la Décision: Méthodes et cas. Paris: Economica, 1993.

[3] Fodor J, Roubens M. Fuzzy preference modelling and multi-criteria decision support. Dordrecht: Kluwer Academic Publishers, 1994.

[4] De Baets B, Van de Walle B, Kerre E. Fuzzy preference structures and their characterization. The Journal of Fuzzy Mathematics 1995;3:373-81.

[5] Bisdorff R, Roubens M. On defining fuzzy kernels from $\mathscr{L}$-valued simple graphs. In: Proceedings of the Conference IPMU'96. Granada, July 1996. p. 593-9.

[6] Kitaïnik L. Fuzzy decision procedures with binary relations: towards a unified theory. Boston: Kluwer Academic Publishers, 1993. 
[7] Bisdorff R, Roubens M. On defining and computing fuzzy kernels from $\mathscr{L}$-valued simple graphs. In: Da Ruan et al., editors. Intelligent Systems and Soft Computing for Nuclear Science and Industry, FLINS'96 Workshop. Singapore: World Scientific Publishers, 1996. p. 113-23.

[8] Bisdorff R. Bi-pole ranking from pairwise fuzzy outrankings. Belgian Journal of Operations Research, Statistics and Computer Science 37:97.

R. Bisdorff vice-president 2 (1997-2000) of EURO, the Federation of European Operational Research Societies within IFORS. He heads the STADE (Statistics \& Decision) unit at the Centre de Recherche Public-Centre Universitaire in Luxembourg. He obtained his Ph.D. degree from the University Paris-Dauphine (France). His main interest is focused on the application of constraint logic programming techniques to multi-valued preference modelling. 